\title{
Linear Viscoelastic and Dielectric Relaxation Response of Unentangled UPy-Based Supramolecular Networks
}

Shabbir, Aamir; Javakhishvili, Irakli; Cerveny, Silvina; Hvilsted, Søren; Skov, Anne Ladegaard; Hassager, Ole; Alvarez, Nicolas J.

Published in:

Macromolecules

Link to article, DOI:

10.1021/acs.macromol.6b00122

Publication date:

2016

Document Version

Peer reviewed version

Link back to DTU Orbit

Citation (APA):

Shabbir, A., Javakhishvili, I., Cerveny, S., Hvilsted, S., Skov, A. L., Hassager, O., \& Alvarez, N. J. (2016). Linear Viscoelastic and Dielectric Relaxation Response of Unentangled UPy-Based Supramolecular Networks.

Macromolecules, 49(10), 3899-3910. https://doi.org/10.1021/acs.macromol.6b00122

\section{General rights}

Copyright and moral rights for the publications made accessible in the public portal are retained by the authors and/or other copyright owners and it is a condition of accessing publications that users recognise and abide by the legal requirements associated with these rights.

- Users may download and print one copy of any publication from the public portal for the purpose of private study or research.

- You may not further distribute the material or use it for any profit-making activity or commercial gain

- You may freely distribute the URL identifying the publication in the public portal 


\title{
Linear Viscoelastic and Dielectric Relaxation Response of Unentangled UPy based Supramolecular Networks
}

Aamir Shabbir, ${ }^{+}$Irakli Javakhishvili, ${ }^{+}$Silvina Cerveny, ${ }^{\ddagger}$ Søren Hvilsted, ${ }^{+}$Anne L. Skov, ${ }^{\dagger}$ Ole Hassager, ${ }^{\dagger}$ and Nicolas J. Alvarez, ${ }^{*} \mathbb{I}$

Department of Chemical and Biochemical Engineering- Kgs. Lyngby 2800 Denmark, Materials

Physics Center-San Sebastian 20018, Spain, and Department of Chemical and Biological Engineering- Philadelphia PA 19104 USA

E-mail: nja49@drexel.edu

\begin{abstract}
Supramolecular polymers possess versatile mechanical properties and a unique ability to respond to external stimuli. Understanding the rich dynamics of such associative polymers is essential for tailoring user defined properties in many products. Linear copolymers of 2-methoxyethyl acrylate (MEA) and varying amounts of 2-ureido-4[1H]-pyrimidone (UPy) quadruple hydrogen-bonding side units were synthesized via free radical polymerization. Their linear viscoelastic response was studied via small amplitude oscillatory shear (SAOS). The measured linear viscoelastic envelope (LVE) resembles that of a well entangled polymer melt with a distinct
\end{abstract}

\footnotetext{
${ }^{*}$ To whom correspondence should be addressed

${ }^{\dagger}$ Technical University of Denmark

‡University of the Basque Country

IIDrexel University
} 
plateau modulus. Dielectric relaxation spectroscopy (DRS) was employed to independently examine the lifetime of hydrogen bond units. DRS reveals a high frequency $\alpha$-relaxation associated with the dynamic glass transition, followed by a slower $\alpha^{*}$ relaxation attributed to the reversible UPy hydrogen bonds. This timescale is referred to as the bare lifetime of hydrogen bonding units. Using the sticky Rouse model and a renormalized lifetime, we predict satisfactorily the LVE response for varying amounts of UPy side groups. The deviation from the sticky Rouse prediction is attributed to polydispersity in the distribution of UPy groups along the chain backbone. We conclude that the response of associating polymers in linear viscoelasticity is general and does not depend on the chemistry of association, but rather on the polymer molecular weight (MW) and MW distribution, the number of stickers per chain, $n_{s}$, and the distribution of stickers along the backbone.

\section{Introduction}

Polymer networks utilizing hydrogen bonding as transient physical interactions hold a prominent place in the field of associating polymers. Although isolated hydrogen bonding interactions are typically not as strong as other non-covalent interactions such as ionic interactions ${ }^{1-6}$ or metal-ligand coordination, ${ }^{7,8}$ their ability to form highly directional and versatile associations make them very useful. ${ }^{9}$ The association strength of an isolated hydrogen bond in a polymer melt depends on the nature of donor and acceptor. Weak hydrogen bonding associations lead to viscoelastic liquids, whereas strong hydrogen bonds result in rubbery like polymer networks. The association energies of hydrogen bonds typically span from one to tens of $k_{B} T$, which makes the nature of these interactions thermally reversible. ${ }^{10}$ At high temperatures, hydrogen bonds become weak leading to Newtonian like fluid properties, whereas at low temperatures they form elastic networks spanning many decades in frequency. ${ }^{11,12}$ Understanding the rich dynamics of such associating polymers offers opportunities for property manipulation and is thus interesting 
from both fundamental and applied standpoints.

The process of breaking and reformation of reversible associations (stickers) controls the dynamics of supramolecular polymers. ${ }^{13}$ Baxandall ${ }^{14}$ and Green and Tobolsky ${ }^{15}$ showed that the stress relaxation via breaking and reformation of reversible associations is essentially Rouse like on timescales larger than the association lifetime. ${ }^{1,14,15}$ Chain reptation can complicate the situation in case of entangled polymer melts. ${ }^{13}$ In this study, our focus is on unentangled polymer melts. The viscoelastic response of unentangled reversible networks near and above the gel point has been described by the modified Rouse model called the sticky Rouse model. ${ }^{16}$ According to this theory, reversible associations act as a second source of friction for the polymer chain thus delaying the terminal relaxation. ${ }^{1,17}$

While a few recent studies have examined the applicability of Green and Tobolsky ideas on ionomers, ${ }^{1}$ very few studies have investigated its applicability in hydrogen bonded systems. Chen et al. were the first to quantitatively describe the LVE response of unentagled poly-ether-ester sulphonate copolymer ionomers using the sticky Rouse model. ${ }^{1}$ Feldman et al., used the theory by Rubinstein and Semenov, which considers a single mode Rouse model for frequencies lower than the inverse association lifetime. ${ }^{12} \mathrm{~A}$ recent paper from Tang et al. has shown the LVE rheological response of protein hydrogels to be dominated by sticky Rouse dynamics. ${ }^{10}$ In their case, the sticky Rouse signature is due to the hindered motion of associative protein molecules in an aggregated state.

The strength and reversibility of hydrogen bonds can be tailored by exploiting multiple hydrogen bond (MHB) arrays. ${ }^{9,18}$ Supramolecular polymers based on multiple hydrogen bonds which exploit the trait of co-operativity have gained large interest recently, showing remarkable properties since they combine directionality and versatility. Since the introduction of 6-methyl-2-ureido-4[1H]-pyrimidone-bearing methacrylate (UPyMA) by Long and co-workers, ${ }^{19}$ several polymers with hydrogen bonding groups have been synthesized. For example, hydrogen bonding copolymers of UPyMA with $n$-butyl acrylate, ${ }^{11,19}$ 2-ethylhexyl methacrylate, ${ }^{20}$ and 2-hydroxyethyl methacrylate ${ }^{21}$ have been syn- 
thesized by making use of either free or controlled radical polymerization method. With thermally tunable strength of association, these hydrogen bonding copolymers display stimuli responsive rheological behavior, and may be employed as shape memory, selfhealing, and adhesive materials.

This paper is a continuing work in the effort to fully understand the dynamics of hydrogen bonding associating polymers. Here, we report the synthesis and characterization of copolymers based on 2-methoxyethyl acrylate (MEA) incorporating UpyMA. More specifically, we examine two copolymers with increasing amount of UPyMA groups: 3 $\%$ UPyMA, and $8 \%$ UPyMA. The percentage of UPyMA groups is determined using NMR spectroscopy. PMEA is chosen due to its FDA approval in medical devices and applications. ${ }^{22}$ The inherent freezing bound water bestows excellent blood compatibility to the intermediately hydrophilic PMEA ${ }^{22}$, and allows its exploitation as coating material for circuits and tubes in cardiopulmonary bypass and catheters for central veins of blood vessels ${ }^{22}$ as well as for artificial oxygenators. ${ }^{23}$ MEA has been employed as one of the building blocks in preparation of random, ${ }^{24}$ block, ${ }^{25,26}$ and graft copolymers ${ }^{27,28}$ by means of controlled radical polymerizations.

In this paper, MEA and UPyMA copolymers are synthesized via free radical polymerization resulting in a random distribution of the UPy (hydrogen bonding) sites. Note that this is in contrast with the polycondensation process where the distance between the sticker groups is strictly controlled by the length of the monomer unit. This study examines the effect of UPyMA density on the copolymer's linear viscoelasticity. The experimental data is compared to the sticky Rouse model considering both polydispersity of the copolymer and polydispersity in the number of stickers per chain. The latter represents a novel analysis to address the open question regarding the effect of associating group dispersion on the terminal regime power-law. The model is parameterized to the fullest extent via experimental data in an effort to minimize adjustable parameters. For example, we independently determine the association lifetime of hydrogen bonds via di- 
electric relaxation spectroscopy (DRS). The original sticky Rouse model is compared to experimental data and is shown to work reasonably well in predicting the viscoelasticity for low density of UPyMA units, it has difficulty at high UPyMA density. The modified sticky Rouse model taking into account polydispersity in the number of stickers works very well in fitting the experimental data via one adjustable parameter, the standard deviation of stickers per chain. The comparison of our results with other studies in the literature suggest that associating groups regardless of association mechanism (i.e. ionic, hydrogen bonding, etc) have three common characteristics in linear viscoelasticity depending on polydispersity of chain molecular weight (MW), number of stickers per chain, and dispersion of stickers.

\section{Experimental Section}

\section{Materials}

2-Methoxyethyl acrylate (MEA; Aldrich, $98 \%$ ) was passed through a short aluminum oxide column (Sigma-Aldrich, activated, basic, Brockmann I, standard grade, $\sim 150$ mesh, $58 \AA$ ) before use. $N, N$-Dimethylformamide (DMF; Sigma-Aldrich, $\geq 99.9 \%$ ) was dried over molecular sieves. $\alpha, \alpha^{\prime}$-Azoisobutyronitrile (AIBN; from Ventron) was recrystallized from methanol. 2-Isocyanatoethyl methacrylate (Aldrich, $98 \%$ ), 2-amino-4-hydroxy-6methylpyrimidine (Aldrich, 98 \%), tetrahydrofuran (THF; Sigma-Aldrich, $99.9 \%$ ), diethyl ether (Sigma-Aldrich, $\geq 99.8 \%$ ), heptane (Sigma-Aldrich, $99 \%$ ), methanol (SigmaAldrich, $99.9 \%$ ), dimethyl sulfoxide (DMSO; SAFC, $\geq 99 \%$ ), $\mathrm{CDCl}_{3}$ (Aldrich, 99.8 atom $\% \mathrm{D}$ ), and DMSO- $d_{6}$ (Aldrich, 99.9 atom\% D) were used as received. 6-Methyl-2-ureido$4[1 H]$-pyrimidone-bearing methacrylate (UPyMA) was synthesized as reported in literature. $^{19}$ 


\section{Analytical Techniques}

NMR experiments were carried out on a Bruker Avance $300 \mathrm{MHz}$ spectrometer. Chemical shifts are reported in ppm.

Molecular weights, MW, and polydispersity indices (PDI) were estimated by size exclusion chromatography (SEC) using an HLC-8320GPC from Tosoh Corporation Bioscience Division equipped with refractive index and UV detectors employing three PFG micro columns (100 $\AA, 1000 \AA$, and $4000 \AA$ ) from Polymer Standards Service (PSS). The samples were run in $\mathrm{DMF}(5 \mathrm{mM} \mathrm{LiCl})$ at $50{ }^{\circ} \mathrm{C}\left(0.3 \mathrm{~mL} \mathrm{~min}^{-1}\right)$. Molecular weights were calculated using WinGPC Unity 7.4.0 software and poly(methyl methacrylate) (PMMA) standards from PSS. The copolymer composition, $F_{U P Y M A}$, and the average number of stickers, $n_{s}$, were estimated from ${ }^{1} \mathrm{H}$ NMR experiments and number average molecular weights estimated by SEC. The molecular weight of a sticky segment, $M_{s}$, was determined using the weight average molecular weights from SEC.

Attenuated total reflectance Fourier transform infrared (ATR FTIR) spectra in the range of 4000-350 $\mathrm{cm}^{-1}$ were recorded on a Nicolet iS50 ATR spectrometer with a diamond crystal from Thermo Scientific.

Thermal transitions were measured in a range of $-90{ }^{\circ} \mathrm{C}$ to $100{ }^{\circ} \mathrm{C}$ at a heating rate of $10^{\circ} \mathrm{C} \mathrm{min}^{-1}$ on a differential scanning calorimeter (DSC) Q1000 from TA. Glass transition temperatures were obtained from the second heating curve. All analytically determined values are tabulated in Table 1.

\section{Synthetic Procedures}

All reactions were carried out under nitrogen flow.

General polymerization protocol. A Schlenk tube was charged with MEA, AIBN, and DMF along with comonomer UPyMA where applicable. The reaction mixture was stirred and deoxygenated by bubbling nitrogen through for $30 \mathrm{~min}$. The tube was then immersed 
in an oil bath at $60^{\circ} \mathrm{C}$, and the polymerization was carried out for $5 \mathrm{~h}$. Afterwards the tube was taken out of the bath, and the reaction mixture was exposed to air. It was then precipitated twice from THF or DMF in diethyl ether-heptane (1:1) mixture. The product was dried in the vacuum oven until no residual solvent was detected by spectroscopic means. Yield: $66-76 \%$.

E.g.: P(MEA-co-UPyMA) 8\%. MEA (2.6 mL, $20.2 \mathrm{mmol})$, UPyMA (215 mg, $0.77 \mathrm{mmol})$, AIBN (16 mg, $0.10 \mathrm{mmol})$, and DMF (15 mL) were taken. SEC: $\bar{M}_{w}=68.6 \mathrm{kDa}, \bar{M}_{w} / \bar{M}_{n}$ = 3.6. IR (neat): 2931, 2885, 2820, 1727, 1662, 1588, 1526, 1448, 1404, 1384, 1333, 1249, 1198, 1160, 1125, 1097, 1028, 983, 952, 861, 764, 601, $532 \mathrm{~cm}^{-1}$. ${ }^{1} \mathrm{H}$ NMR (300 MHz, DMSO-d $d_{6}$,

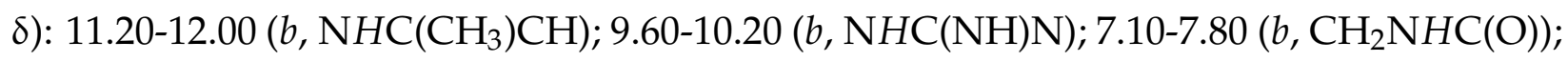
$5.77\left(s, \mathrm{C}(\mathrm{O}) \mathrm{CHC}\left(\mathrm{CH}_{3}\right) \mathrm{NH}\right) ; 3.85-4.32\left(b, \mathrm{OCH}_{2} \mathrm{CH}_{2} \mathrm{OC}(\mathrm{O})\right.$ and $\left.\mathrm{NHCH}_{2} \mathrm{CH}_{2} \mathrm{OC}(\mathrm{O})\right)$; 3.383.65 (b, $\mathrm{OCH}_{2} \mathrm{CH}_{2} \mathrm{OC}(\mathrm{O})$ and $\left.\mathrm{NHCH}_{2} \mathrm{CH}_{2} \mathrm{OC}(\mathrm{O})\right)$; 3.14-3.30 (b, $\left.\mathrm{CH}_{3} \mathrm{O}\right)$; 2.15-2.43 (b, $\mathrm{CH}_{2} \mathrm{CHC}(\mathrm{O}) \mathrm{O} ; 2.10\left(s, \mathrm{C}(\mathrm{O}) \mathrm{CHC}\left(\mathrm{CH}_{3}\right) \mathrm{NH}\right) ; 0.75-1.95\left(b, \mathrm{CH}_{2} \mathrm{CHC}(\mathrm{O}) \mathrm{O}, \mathrm{CH}_{2} \mathrm{C}\left(\mathrm{CH}_{3}\right) \mathrm{C}(\mathrm{O}) \mathrm{O}\right.$, and $\left.\mathrm{CH}_{2} \mathrm{C}\left(\mathrm{CH}_{3}\right) \mathrm{C}(\mathrm{O}) \mathrm{O}\right)$.

\section{LVE Measurements}

Linear viscoelasticity for the three P(MEA-co-UPyMA) polymers was measured via SAOS performed with an ARES-G2 (TA instruments) rheometer. Temperature was controlled using a convection oven operating in nitrogen. $8 \mathrm{~mm}$ diameter parallel plate geometry was used for all experiments. No slippage problem was observed due to good adhesion of the sample and the plate geometry. For PMEA, LVE segments were measured between -30 ${ }^{\circ} \mathrm{C}$ and $100{ }^{\circ} \mathrm{C}$. For P(MEA-co-UPyMA) 3\%, LVE segments were measured between -25 ${ }^{\circ} \mathrm{C}$ and $100{ }^{\circ} \mathrm{C}$. For P(MEA-co-UPyMA) 8\%, LVE segments were measured between -20 ${ }^{\circ} \mathrm{C}$ and $120^{\circ} \mathrm{C}$. When possible, time temperature superposition was used to create master curves. Vertical shift factors, $\beta$, are given in Table 1 of SI. Since instrument compliance is known to influence the LVE data in the glassy regime $\left(10^{8} \mathrm{~Pa}\right)$, measurements were corrected following the method reported by Schröter et al. for $8 \mathrm{~mm}$ plates on an ARES- 
G2 rheometer. ${ }^{29}$

\section{DRS Measurements}

To measure the complex dielectric permittivity, $\epsilon^{*}(\omega)=\epsilon^{\prime}(\omega)-i \epsilon^{\prime \prime}(\omega)$, we have used a Novocontrol Alpha-Analyzer with a Quatro Cryosystem temperature control. Each sample was melted on the dielectric electrodes with a Teflon spacer of $0.1 \mathrm{~mm}$. The sample diameter was $30 \mathrm{~mm}$. The dielectric measurements were done approximately from $\left(T_{g}+\right.$ $90 \mathrm{~K})$ to $\left(T_{g}-10 \mathrm{~K}\right)$

\section{Results}

Figure 1 depicts the structure of the copolymer P(MEA-co-UPyMA).

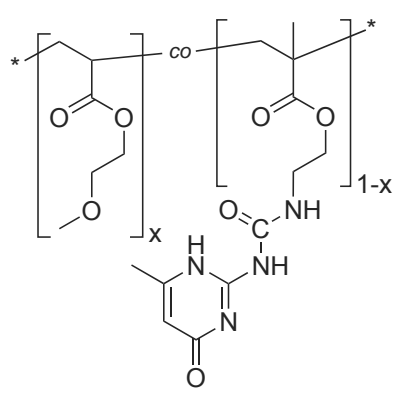

Figure 1: Copolymer of MEA and UPyMA

Together with the homopolymer, PMEA, two copolymers with varying UPyMA content have been synthesized by adjusting the monomer feed ratio in free radical polymerization. Formation of the copolymer has been verified by FTIR spectroscopy, which reveals absorption bands at $1727 \mathrm{~cm}^{-1}, 1662 \mathrm{~cm}^{-1}, 1588 \mathrm{~cm}^{-1}$ that are attributed to ester, urea, and pyrimidone groups, respectively (Figure 2). 


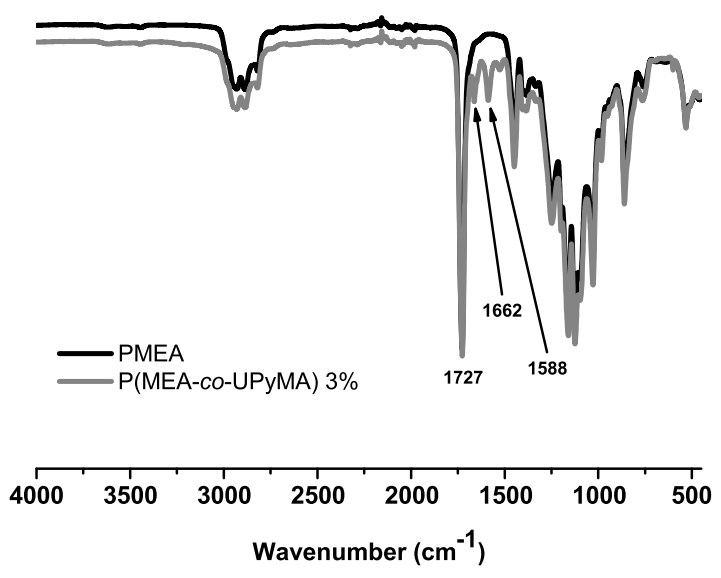

Figure 2: Overlay of FTIR spectra of PMEA and P(MEA-co-UPyMA)3\%

The band at $1588 \mathrm{~cm}^{-1}$ is characteristic to the dimerized UPy, which implies that the presence of the multitude of methoxyethyl fragments along the polymeric backbone does not disrupt the hydrogen bond formation. ${ }^{21,30}$ The copolymer composition is further elucidated by NMR spectroscopy. ${ }^{1} \mathrm{H}$ NMR spectroscopy experiments corroborate incorporation of the UPyMA repeating units; resonance signals 10 and $\mathbf{1 1}$ at $5.77 \mathrm{ppm}$ and 2.10 ppm, respectively, are unambiguously ascribed to the methine and methyl groups of the pyrimidone fragment (Figure 3). A heteronuclear single quantum coherence (HSQC) experiment substantiates the assignment revealing the coupled carbons at $104 \mathrm{ppm}$ and 23 ppm, respectively. The UPyMA molar fractions in the two copolymers are estimated to be $3 \%$ and $8 \%$, respectively. This estimation is made by comparing the integrals of the resonance signals 2 and 5 in Figure 3, attributed to the MEA repeating unit, with the integrals of the overlapping peaks 3, 8 and 4, 9 originating from the MEA and UPyMA repeating units. 


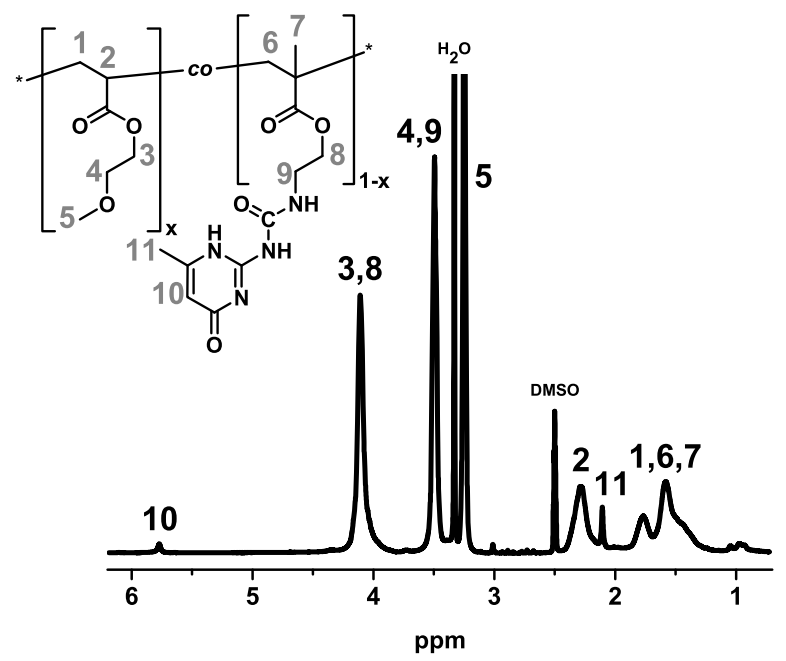

Figure 3: ${ }^{1} \mathrm{H}$ NMR spectrum of P(MEA-co-UPyMA) $8 \%$ acquired in DMSO- $d_{6}$

The weight average molecular weights $\left(\bar{M}_{w}\right)$ and molecular weight distributions (MWD) of the homo- and copolymers have been obtained by SEC. SEC measurements show a PDI $\geq 2.9$, which is intrinsic to free radical polymerizations.

Influence of the content and nature of the H-bonding units on the $T g$ has been probed by DSC. $\mathrm{Tg}$ increases from $-35^{\circ} \mathrm{C}$ for PMEA to $-31^{\circ} \mathrm{C}$ and $-28^{\circ} \mathrm{C}$ for P(MEA-co-UPyMA) 3\% and P(MEA-co-UPyMA) 8\%, respectively. Characteristics of the homo- and copolymers are summarized in Table 1.

Table 1: Molecular weight characteristics, composition, and thermal properties of homoand copolymers

$\begin{array}{ccccccc}\text { Polymer } & \begin{array}{c}M_{w}{ }^{a} \\ {[\mathrm{kDa}]}\end{array} & \text { PDI }^{a} & \begin{array}{c}F_{U P y M A}{ }^{b} \\ {[\mathrm{~mol} \%]}\end{array} & \begin{array}{c}\text { [Stickers/chain] } \\ n_{s}\end{array} & \begin{array}{c}T_{g} \\ { }^{\circ} \mathrm{C}\end{array} & \begin{array}{c}M_{s}{ }^{b} \\ \mathrm{kDa}\end{array} \\ \text { PMEA } & 65.3 & 2.9 & 0 & 0 & -35 & - \\ \text { P(MEA-co-UPy MA) 3\% } & 32.1 & 2.9 & 3.0 & 2 & -31 & 12.9 \\ \text { P(MEA-co-UPy MA) 8\% } & 68.6 & 3.6 & 8.0 & 11 & -28 & 6.4 \\ { }^{a} \text { Obtained by SEC; }{ }^{b} \text { estimated by combining results from }{ }^{1} \text { H NMR spectroscopy and } \\ \text { SEC. }\end{array}$




\section{Linear Viscoelasticity}

Master curves representing linear viscoelasticity for all three samples are presented in Figure 4. More specifically, Figure 4 shows $G^{\prime}(\omega)$ and $G^{\prime \prime}(\omega)$ for PMEA, P(MEA-co-UPyMA) $3 \%$, and P(MEA-co-UPyMA) $8 \%$ as a function of frequency, $\omega$, at $T_{r e f}=25^{\circ} \mathrm{C}$. The rheological response of PMEA shows a power-law dependence of $G^{\prime}$ and $G^{\prime \prime}$ at relatively high frequency, followed by a terminal regime at relatively low frequency (i.e. $G^{\prime}$ and $G^{\prime \prime}$ of powerlaw 1 and 2, respectively). This is the typical response of an unentangled polymer melt and is typically referred to as Rouse dynamics in the literature. Also in Figure 4, the rheological response of UPyMA containing copolymers is represented in colored symbols. The presence of hydrogen bonds produces a substantial change in the measured linear viscoelastic response. At moderate frequencies, a discernible plateau modulus in $G^{\prime}(\omega)$ is noted which spans approximately four decades in frequency. The width of the rubbery plateau increases with the number of UPy side groups. Furthermore, the magnitude of the rubbery plateau also increases with the number of UPy side groups suggesting the strength of the network to be a function of concentration of hydrogen bonds. At low frequencies, there is an evident deviation from terminal power law slopes for UPyMA containing copolymers. This observation has been previously observed in LVE of several hydrogen bonding associating polymers. $6,11,12,31,32$ This could be due to either, (i) the relatively large PDI of the polymer, (ii) the polydispersity in UPy side group spacing along the backbone, or (iii) the signature response of hydrogen bonding polymers. Although concrete evidence is lacking, we hypothesize that the lack of a terminal response is most likely due to (ii), the polydisperse UPy side group spacing along the backbone. 


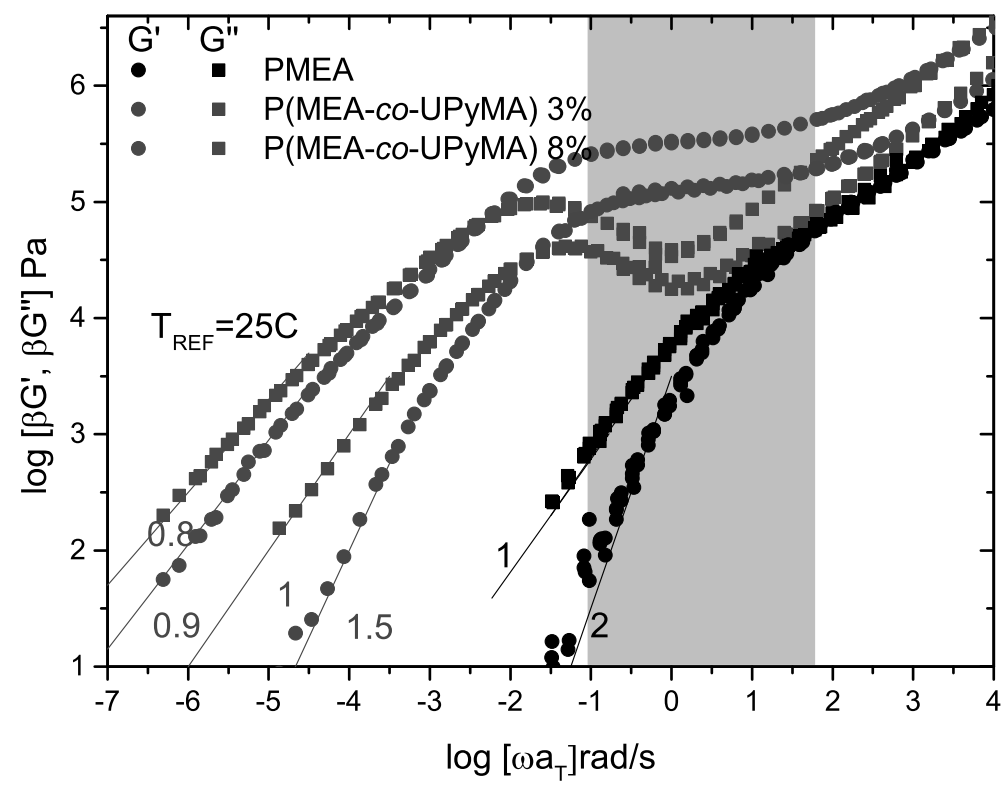

Figure 4: Comparison of linear viscoelastic master curves of PMEA and P(MEA-coUPyMA) systems shifted to $T_{r e f}=25^{\circ} \mathrm{C}$. The gray region represents the accessible time frame of a single experiment

The time temperature superposition (TTS) shift factors $a_{T}$ are shown in Figure 5. As expected, PMEA follows typical Williams-Landel-Ferry (WLF) behavior. Shift factors for the two UPyMA containing polymers show a transition from WLF to Arrhenius-like behavior as a function of temperature. More specifically, WLF like behavior at low temperatures and Arrhenius-like behavior at higher temperatures. The transition to Arrhenius behavior is suggestive of kinetics of association/disassociation dynamics of hydrogen bonds. At low temperatures the hydrogen bonds can be thought of as being static. However at temperatures above which the thermal energy $\left(k_{B} T\right)$ becomes comparable to the activation energy of hydrogen bond disassociation, the hydrogen bonds are dynamic. The data was plotted as suggested by Liu et al. in order to see a second transition corresponding to $T_{g}$, however the temperature range of the LVE data was not low enough to observe one. ${ }^{33}$

For the two UPyMA copolymers, the gray shaded region in Figure 4 signifies the fail- 
ure in time-temperature-superposition (TTS). We hypothesize that the breakdown of TTS is due to a competition of timescales, one for each molecular mechanism: (i) the dynamics of the polymer chain, and (ii) the fluctuations of hydrogen bonds. At $25^{\circ} \mathrm{C}$, both polymer chain dynamics (denoted by PMEA LVE) and hydogen bond fluctuations (denoted by rise in plateau modulus) occur in the same frequency range. The breakdown of TTS is most likely related to the coinciding of these two mechanisms occurring in the same frequency range. Thermorheological complexibility has been observed for other hydrogen bonding associating polymers ${ }^{11,34,35}$ and ionomers. ${ }^{1-4}$

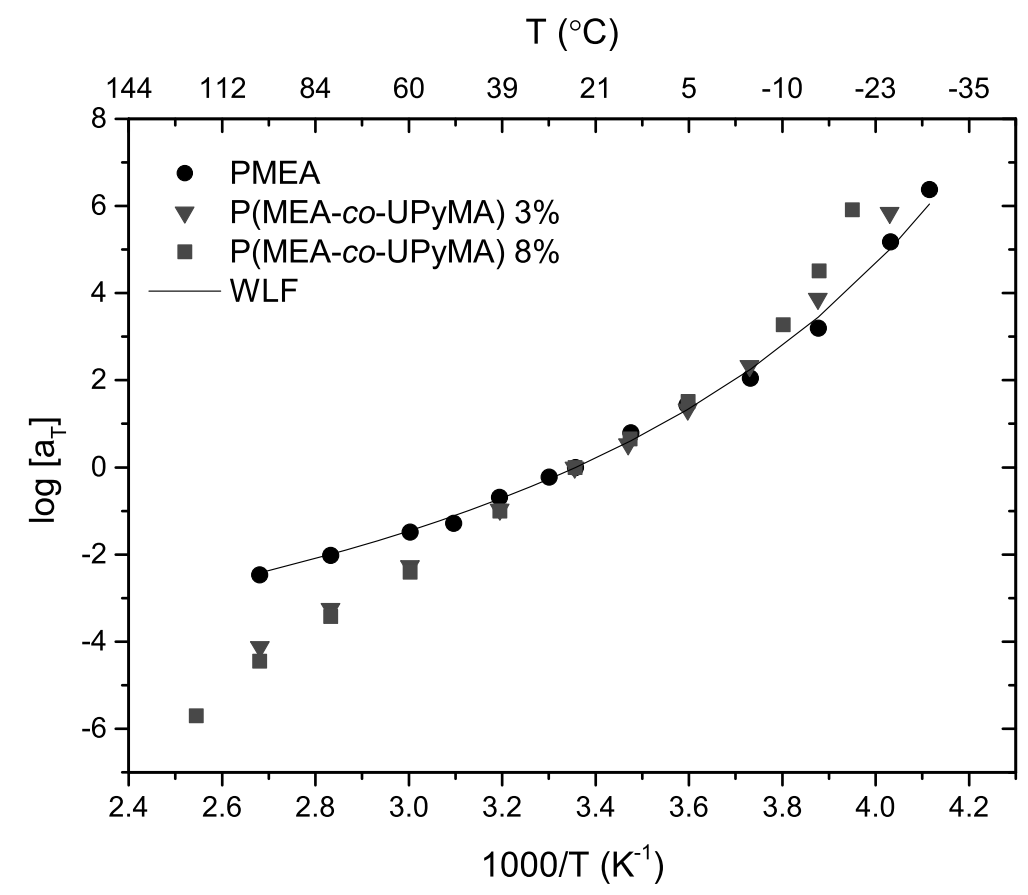

Figure 5: Temperature dependence of apparent shift factors for PMEA and P(MEA-coUPyMA) systems shifted to $T_{r e f}=25^{\circ} \mathrm{C}$

The activation energies, $E_{a}$, calculated from Arrhenius relationship:

$$
\ln a_{T}=-\frac{E_{a}}{R T}+C
$$

are 110 and $125 \mathrm{~kJ} / \mathrm{mol}$ for P(MEA-co-UPyMA) 3\% and P(MEA-co-UPyMA) 8\% re- 
spectively.

\section{Dielectric relaxation spectroscopy (DRS)}

Broadband dielectric spectroscopy is a technique based on the interaction of an external field with the electric dipole entities in the sample. ${ }^{36}$ The relaxation phenomena are related to molecular fluctuations of dipoles due to the movements of molecules or their parts in a potential field. Additional contributions to the observed relaxation can be caused by motion of mobile charge carriers and polarization of the electrode itself. If the frequency of the applied oscillatory field corresponds to reorientation times, $\tau$, of the molecular dipoles, there is a characteristic response of the storage permittivity, $\epsilon^{\prime}$, and loss permittivity, $\epsilon^{\prime \prime} . \epsilon^{\prime}(\omega)$ decreases with increasing $\omega$ depicting a characteristic step, while $\epsilon^{\prime \prime}(\omega)$ exhibits a maximum with increasing $\omega$. The essential quantity, $\tau$ can be extracted from this behavior. The frequency corresponding to the maximum loss, $v_{p}$, is related to the characteristic relaxation time, $\tau=1 / 2 \pi v_{p}$. $\epsilon^{\prime}$ and $\epsilon^{\prime \prime}(\omega)$ for PMEA are shown in Figure 6 for varying temperature. It is evident from this data that PMEA shows a single characteristic relaxation time (i.e. one maximum in $\epsilon^{\prime \prime}$ and one step-like shoulder in $\epsilon^{\prime}$ ) for the measured frequency range and the temperatures measured. This single and primary relaxation process is referred to as the $\alpha$-relaxation process and is attributed to segmental motion of the chain near the glass transition. 


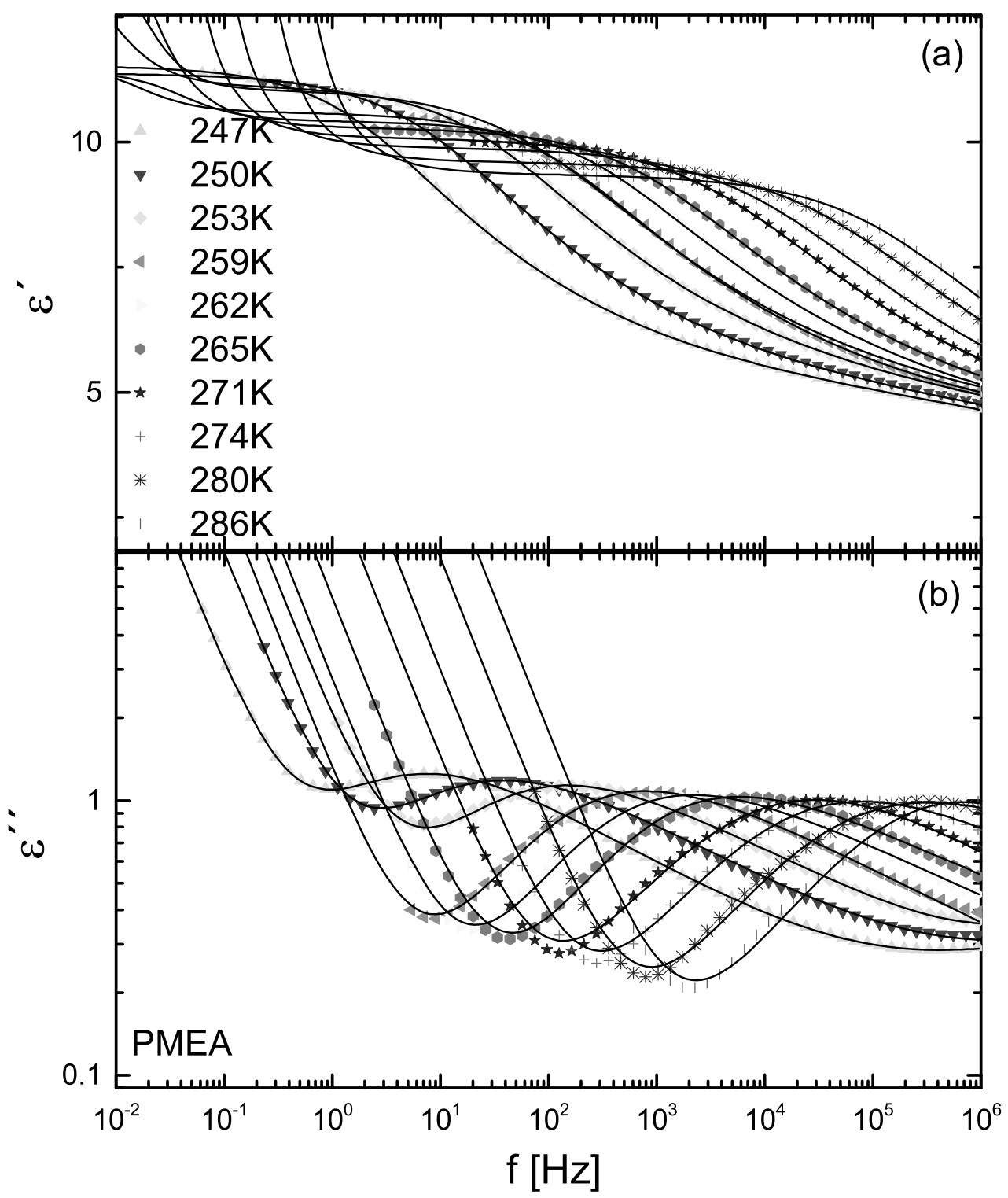

Figure 6: The storage permittivity (a) $\epsilon^{\prime}$ and (b) loss permittivity $\epsilon^{\prime \prime}$ as a function of frequency for pure PMEA (no UPy groups). Note that only one step-like shoulder and one maximum are observed in (a) and (b), respectively, indicating a single relaxation time in this frequency and temperature range. This characteristic relaxation time is denoted as the $\alpha$-relaxation time. The lines represent the best fit to the data using Eqn. 3, which is used to quantitatively determine the relaxation timescale.

Figure $7(\mathrm{a}-\mathrm{c})$ shows both the storage and loss permittivity as a function of frequency and derivative formalism for P(MEA-co-UPyMA) $8 \%$ at different temperatures above $T_{g}$. The real part of the complex permittivity, $\epsilon^{\prime}(\omega)$ (Figure 7a) shows two steps indicating 
two relaxation processes. However, only one relaxation process is observed in $\epsilon^{\prime \prime}(\omega)$, as shown in Figure $7 \mathrm{~b}$. This is due to a strong electrode polarization and/or ionic conductivity which masks $\epsilon^{\prime \prime}$ in the frequency regime of interest. To overcome the issue observed in loss permittivity due to electrode polarization and/or ionic conductivity, it is standard procedure to use the derivative analysis of $\epsilon^{\prime}: 1,36,37$

$$
\epsilon_{d e r}(\omega)=-\frac{2}{\pi} \frac{\partial \epsilon^{\prime}(\omega)}{\partial \log \omega}
$$

The derivative analysis for P(MEA-co-UPyMA) is shown in Figure 7c. From this analysis, it is clear that two relaxation processes are occurring (i.e. two maximums observed). The fast process is related to the glass transition and therefore referred to as $\alpha$-relaxation. The slow process, not present in pure PMEA, is presumably due to hydrogen bonds and is referred to as the $\alpha^{*}$-relaxation in Figure 7c.

Although one can crudely determine the frequency corresponding to the maximum in $\epsilon_{d e r}$, a more quantitative approach is to fit a model to the permittivity data. In this case, the complex dielectric permittivity is described by the superposition of a HavriliakNegami function, two Cole-Cole functions, ${ }^{38}$ and a dc conductivity term. The total fit function was given by

$$
\epsilon^{*}(\omega)=\epsilon_{\infty}+\frac{\Delta \epsilon}{\left[1+\left(i \omega \tau_{0}\right)^{\alpha}\right]^{\beta}}+\sum_{j=1}^{2} \frac{\Delta \epsilon_{j}}{1+\left(i \omega \tau_{j}\right)^{\alpha_{j}}}+i\left(\frac{\sigma_{0}}{\epsilon_{0} \omega}\right)
$$

where $\Delta \epsilon$ is the dielectric strength $\left(\Delta \epsilon=\epsilon_{s}-\epsilon_{\infty}, \epsilon_{\infty}\right.$ and $\epsilon_{s}$ are the unrelaxed and relaxed values of the dielectric constant respectively), $\tau_{0}$ is the relaxation time for the $\alpha$ relaxation mechanism, and $\tau_{j}$ corresponds to the electrode polarization $(j=1)$ and $\alpha^{*}$ relaxation $(j=2), \alpha$ and $\beta$ are the shape parameters which describe the symmetric and the asymmetric broadening of the spectrum, and $\omega=2 \pi f$ is the angular frequency. $\epsilon_{0}$ denotes the vacuum permittivity and $\sigma$ is the static ionic conductivity. Both CC functions were used to describe the $\alpha^{*}$-relaxation and the electrode polarization observed in $\epsilon^{\prime}(\omega)$ (EP) 
respectively, whereas the $\mathrm{HN}$ function takes into account the $\alpha$-relaxation. An example of the fitting procedure is shown in Figure S1 of SI. The shape parameters, $\alpha$ and $\beta$ in equation are approximately $\alpha \approx 0.5$ and $\beta \approx 0.4$ for the $\alpha$-relaxation whereas for $\alpha^{*}$-relaxation, $\alpha$ parameter is close to 0.95 and therefore almost Debye-like. The relaxation strength of both relaxations, $\alpha$ and $\alpha^{*}$-relaxations are shown in Figure S2 of SI. 


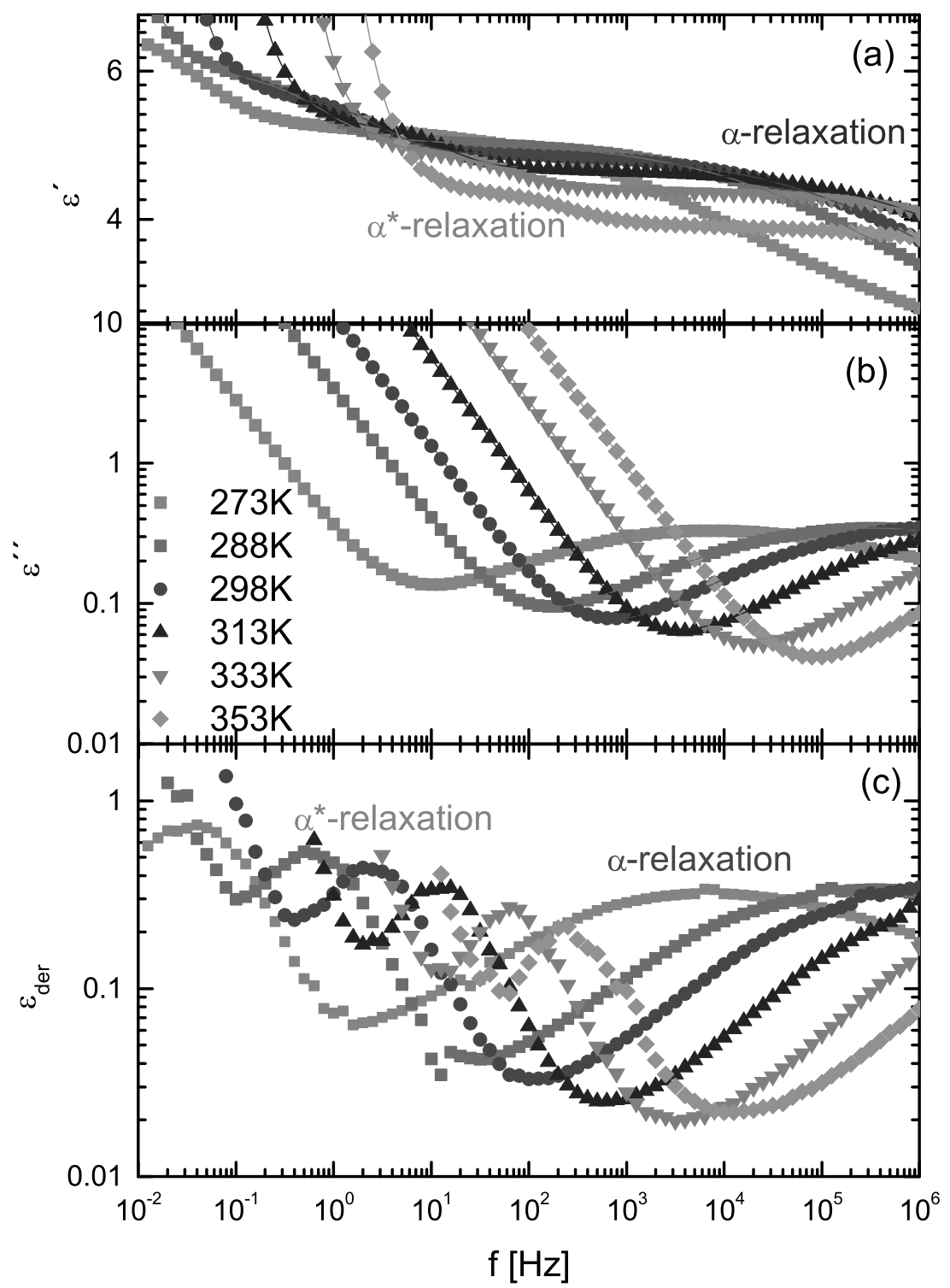

Figure 7: (a) storage permittivity, (b) loss permittivity and (c) derivative formalism spectra for P(MEA-co-UPyMA) $8 \%$. Solid lines through the data points represent the fits to the experimental data using Eqn. 3

The most relevant parameter derived from the fitting procedure is the relaxation time $\tau$, describing the dynamical behavior of the polymeric system. Relaxation processes usu- 
ally follow the empirical Vogel-Fulcher-Tammann (VFT) equation, ${ }^{39,40}$

$$
\tau(\alpha)=\tau_{H} \exp \left(\frac{D T_{0}}{T-T_{0}}\right)
$$

where $\tau_{H}$ is the high temperature limit of the relaxation time, $T_{0}$ is the Vogel temperature, and $D$ is the strength parameter inversely related to the fragility. On the other hand, for a thermally activated behavior, the temperature dependence of the relaxation time follows the Arrhenius law.

The temperature dependence of the relaxation times $\tau_{0}$, and $\tau_{2}$ are plotted in Figure 8 for the three samples in order to determine the dominating relaxation mechanisms for P(MEA-co-UPyMA) systems. As expected, the $\alpha$-relaxation (fast times, high frequency) is well described by the VFT equation. Note that the temperature values at which the relaxation times reach $100 \mathrm{~s}$, i.e., the dielectric $T_{g, 100 s}$ show a good agreement with the corresponding calorimetric values for all the investigated samples (see Table 2 in SI), supporting the idea that this relaxation corresponds with the glass transition timescale. On the other hand, $\tau_{2}$ is well described by the Arrhenius equation suggesting that this process is kinetic in nature. In other words, the molecular relaxation process characterized by $\tau_{2}$ is most likely due to hydrogen bond dynamics. This interpretation is inline with previous studies of UPy containing supramolecular polymers ${ }^{41}$ and other similar systems. ${ }^{42}$ All parameters are tabulated in Table 2 of SI. 


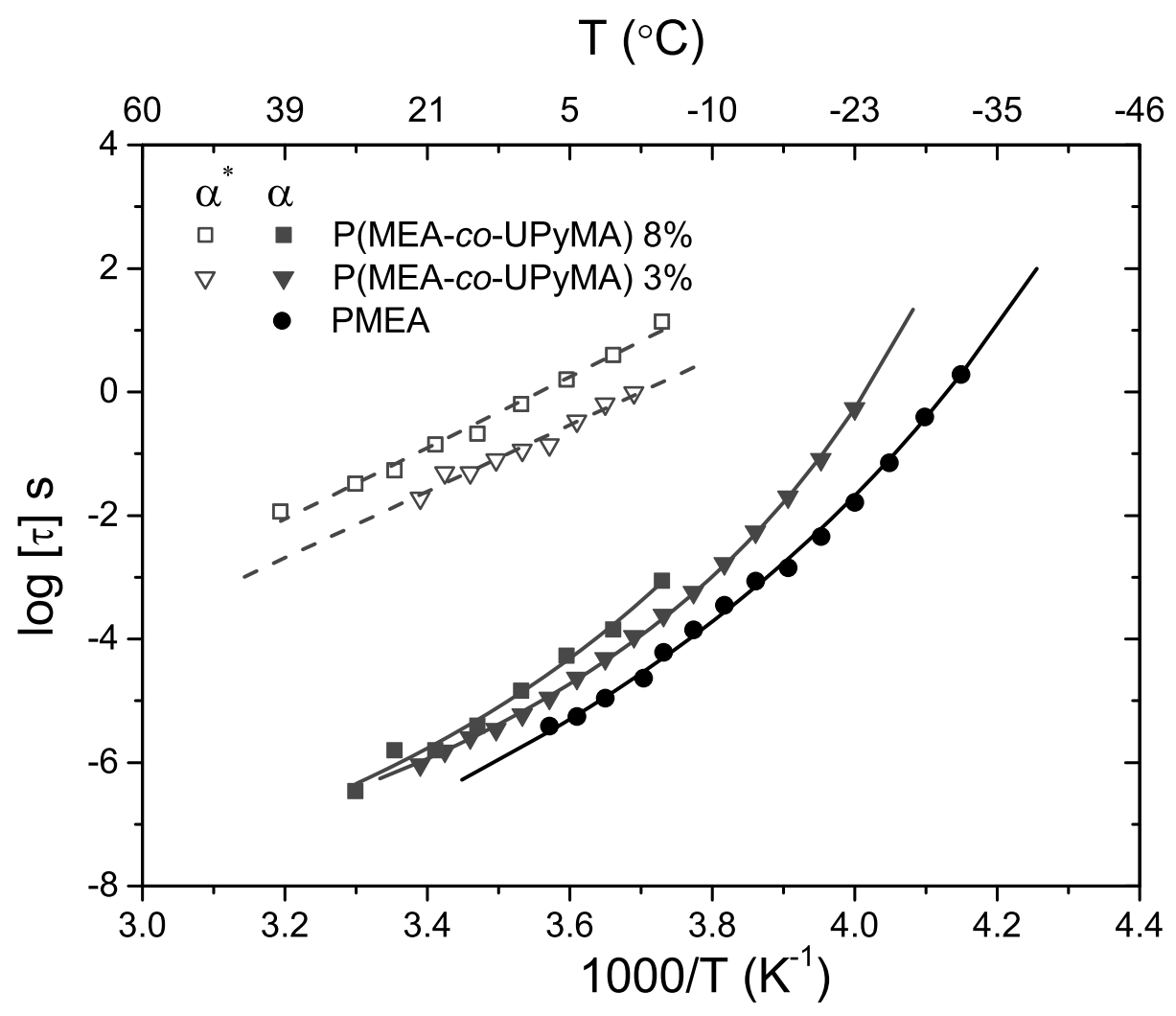

Figure 8: Extracted relaxation times for PMEA and P(MEA-co-UPyMA) polymers as a function of inverse temperature. The timescale associated with $\alpha^{*}$-relaxation shows a distinct powerlaw, indicative of Arrhenius behavior (dotted lines), while timescale associated with $\alpha$-relaxation follows the VFT model (solid lines).

\section{Discussion}

The concept of chain relaxation via repetitive breaking and reforming of reversible bonds along the backbone dates back to early work from Green and Tobolsky. ${ }^{15}$ This concept was extended by Rubinstein and Semenov in their theoretical work on unentangled associating polymer solutions. ${ }^{16}$ Recently Colby and coworkers have experimentally tested these ideas on ionomer melts. ${ }^{1}$ We are interested in testing the sticky Rouse model on the LVE of unentangled polymer melts with hydrogen bonding. The sticky Rouse model first proposed by Rubinstien, Semenov, Leibler and Colby assumes that the normal Rouse modes (higher order modes, $p>N_{s}$, where $N_{s}$ is the number of sticky Rouse segments 
per chain) exist for length scales up to the order of the distance between two associations, known as the sticky Rouse segment. However, the chain dynamics on length scales larger than the length of a sticky Rouse segment is only controlled by the association lifetime, $\tau_{s}$. Thus, the lower order Rouse modes $\left(p \leq N_{s}\right)$ are delayed. The Rouse relaxation modulus taking into account a known molecular weight distribution is given in the time domain as: ${ }^{1,43}$

$$
G_{R}(t)=\sum_{i} \frac{\rho w_{i} R T}{M_{i}} \sum_{p=1}^{N_{i}} \frac{-t p^{2}}{\tau_{R, 0} N_{i}^{2}}
$$

The first summation term takes into account polydispersity in molecular weight, which can be determined independently using SEC. Eq. 4 in Chen et al.considering Eq. 5 can be converted to the frequency domain using a Fourier transform: ${ }^{1}$

$$
\begin{aligned}
& G_{\text {stickyRouse }}^{\prime}(\omega)=\sum_{i} \frac{\rho w_{i} R T}{M_{i}}\left(\sum_{p=1}^{N_{s}} \frac{\left(\tau_{s} N_{s}^{2} p^{-2} \omega\right)^{2}}{1+\left(\tau_{s} N_{s}^{2} p^{-2} \omega\right)^{2}}+\sum_{p=N_{s}+1}^{N_{i}} \frac{\left(\tau_{R, 0} N_{i}^{2} p^{-2} \omega\right)^{2}}{1+\left(\tau_{R, 0} N_{i}^{2} p^{-2} \omega\right)^{2}}\right) \\
& G_{\text {stickyRouse }}^{\prime \prime}(\omega)=\sum_{i} \frac{\rho w_{i} R T}{M_{i}}\left(\sum_{p=1}^{N_{s}} \frac{\left(\tau_{s} N_{s}^{2} p^{-2} \omega\right)}{1+\left(\tau_{s} N_{s}^{2} p^{-2} \omega\right)^{2}}+\sum_{p=N_{s}+1}^{N_{i}} \frac{\left(\tau_{R, 0} N_{i}^{2} p^{-2} \omega\right)}{1+\left(\tau_{R, 0} N_{i}^{2} p^{-2} \omega\right)^{2}}\right)
\end{aligned}
$$

where $w_{i}$ and $M_{i}$ are weight fraction and molecular weight of the $i$ th component, respectively. $\tau_{R, 0}$ is the relaxation time of the elementary Rouse segment, $\tau_{s}$ is the relaxation time of the sticky segment, $N_{i}=M_{i} / M_{0}$ is the number of elementary Rouse segments per chain and $N_{s, i}=M_{i} / M_{s}$ is the number of sticky Rouse segments per chain. $M_{0}$ and $M_{s}$ are the weight average molecular weight of the elementary Rouse segment and sticky Rouse segment, respectively. $M_{s}$ is defined as the ratio of the weight average molecular weight estimated by SEC divided by the average number of stickers determined from NMR spectroscopy.

While Eqs. 6 and 7 take into account polydispersity in molecular weight, data in the literature suggests that dispersity in the distribution of associating groups along the polymer backbone are responsible for shallowing of the low frequency power-law. ${ }^{44}$ In Eqs. 6 and $7 N_{s}$ for a given $M_{i}$ is assumed a single value equal to that determined from NMR. 
In reality, there is a distribution of $N_{s}$ per chain due to the uncontrolled free radical polymerization of the two monomers used in this work. The discrete distribution of $N_{S}$ is potentially represented by a binomial distribution determined by the number of monomer units per chain, $n_{c}=M_{i} / M_{m}$, where $M_{m}$ is the molecular weight of the MEA repeat unit, and the probability of an UPy group adding to a growing chain is defined as, $p=n_{s} / n_{c}$ where $n_{s}$ is the number of stickers per chain determined from NMR reported in Table 1. Application of the binomial distribution to $N_{s}$ leads to a discrete distribution represented by:

$$
\sum_{j}^{n_{c}} f_{j} N_{s, j}=n_{s}
$$

Eqns. 9 and 10 are altered to take the distribution of sticky Rouse groups per chain into account via:

$$
\begin{aligned}
& G_{\text {stickyRouse }}^{\prime}(\omega)=\sum_{i} \frac{\rho w_{i} R T}{M_{i}} \sum_{j} f_{j}\left(\sum_{p=1}^{N_{s, j}} \frac{\left(\tau_{s} N_{s, j}^{2} p^{-2} \omega\right)^{2}}{1+\left(\tau_{s} N_{s, j}^{2} p^{-2} \omega\right)^{2}}+\sum_{p=N_{s, j}+1}^{N_{i}} \frac{\left(\tau_{R, 0} N_{i}^{2} p^{-2} \omega\right)^{2}}{1+\left(\tau_{R, 0} N_{i}^{2} p^{-2} \omega\right)^{2}}\right) \\
& G_{\text {stickyRouse }}^{\prime \prime}(\omega)=\sum_{i} \frac{\rho w_{i} R T}{M_{i}} \sum_{j} f_{j}\left(\sum_{p=1}^{N_{s, j}} \frac{\left(\tau_{s} N_{s, j}^{2} p^{-2} \omega\right)}{1+\left(\tau_{s} N_{s, j}^{2} p^{-2} \omega\right)^{2}}+\sum_{p=N_{s, j}+1}^{N_{i}} \frac{\left(\tau_{R, 0} N_{i}^{2} p^{-2} \omega\right)}{1+\left(\tau_{R, 0} N_{i}^{2} p^{-2} \omega\right)^{2}}\right)
\end{aligned}
$$

The characteristic modulus corresponding to the sticky Rouse segments is defined as $k_{B} T$ per sticker and is given as $G_{c}=P_{0} k_{B} T$, where $P_{0}=\rho N_{A} / M_{s}$ is the number density of stickers, $k_{B}$ is the Boltzmann constant, $\rho$ is the mass density and $N_{A v}$ is the Avogadro number. The corresponding timescale is the sticky Rouse segment time or the sticker lifetime and is evaluated as $\tau_{s} \sim 1 / \omega_{c}$, where $\omega_{c}$ is the characteristic frequency at which $G^{\prime}\left(\omega_{c}\right)=P_{0} k_{B} T .{ }^{1}$ A comparison of the mean sticker lifetime extracted from SAOS rheology $\left(\tau_{s} \sim 1 / \omega_{c}\right)$ and DRS (peak corresponding to $\alpha^{*}$-relaxation process) is shown in Figure 9 a. A fairly good agreement is found between the two techniques. The activation 
energies obtained using DRS and rheology are also comparable.

The gel point represents the point at which physical junctions percolate over the system. ${ }^{45}$ According to the sticky Rouse model above the gel point, multiple associations and disassociations of a given pair of stickers are required before the sticker finds another partner different from its previous partner. This leads to an effective lifetime of the sticker, $\tau_{s}^{*}$. As a consequence this renormalized lifetime, $\tau_{s}^{*}$, is larger than the bare sticker lifetime, $\tau_{s} .{ }^{13,16}$ The mean-field prediction of the gel point, $p_{c}$, for P(MEA-co-UPyMA) $3 \%$ is estimated to be around $1 \mathrm{~mol} \%$ of UPyMA content, while in case of P(MEA-co-UPyMA) $8 \%$ it is around $0.7 \mathrm{~mol} \%$. This implies that the content of the UPyMA repeating units in either copolymer is above the gel point. Therefore, $\tau_{s}^{*}$ will be used in the sticky Rouse model to capture the LVE response. As previously done in other studies, $\tau_{s}^{*}$ is determined from the frequency at which the storage modulus has dropped $10 \%$ of the plateau modulus. ${ }^{12}$ There should be a linear relationship between the timescale measured using DRS, the bare sticker lifetime, $\tau_{s}$, and the effective lifetime $\tau_{s}^{*}$ whose slope corresponds to the proportionality constant between them. In Figure 9 b, there is a clear linear relationship between $\tau_{s}$ and $\tau_{s}^{*} . \tau_{s}^{*}$ is larger than $\tau_{s}$ by a factor of $\sim 40$ for P(MEA-co-UPyMA) $3 \%$, and by a factor of $\sim 25$ for P(MEA-co-UPyMA) 8\%. Surprisingly, the proportionality constant decreases with increasing UPy content, which has no explanation at present. 

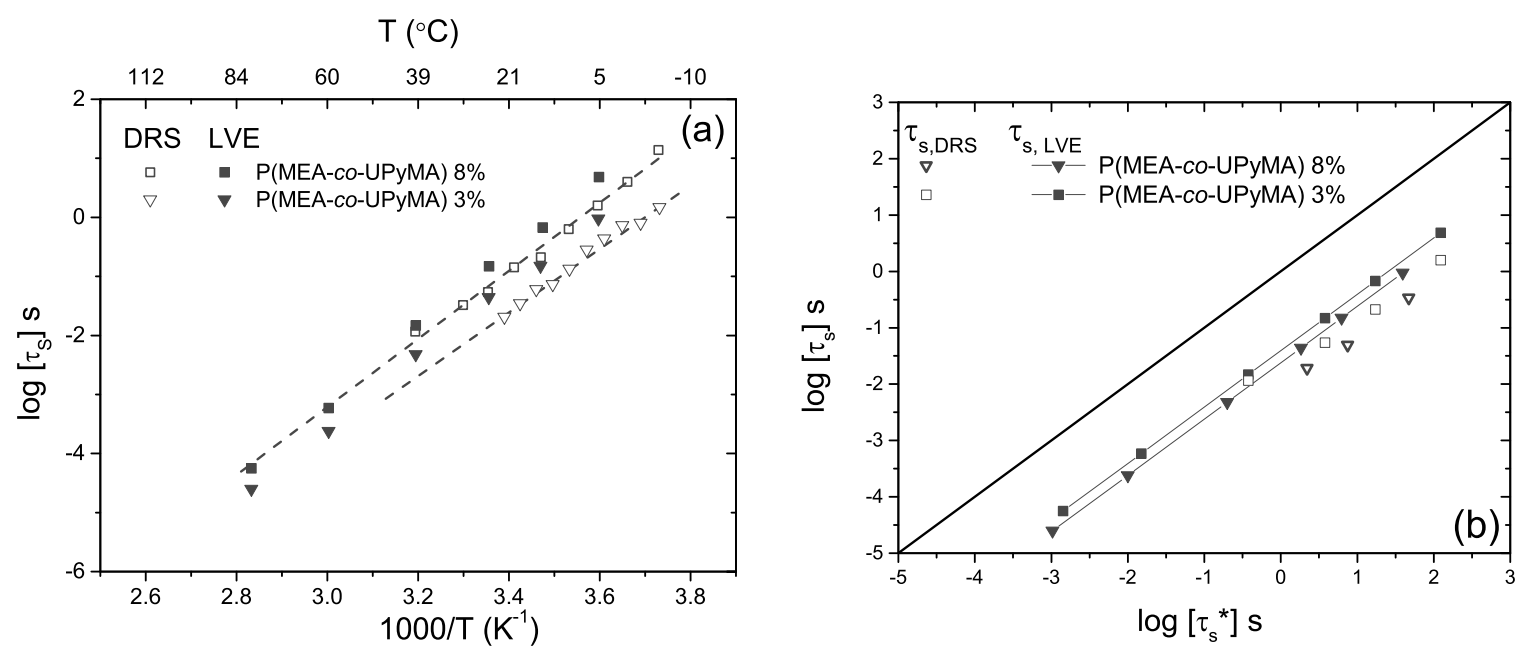

Figure 9: Comparison of (a) bare lifetime of hydrogen bonds obtained using dielectric relaxation spectroscopy / rheology as a function of inverse temperature, and (b) bare lifetime with renormalized lifetime of hydrogen bonds. The solid line represents hypothetical situation in which bare lifetime and renormalized time is equal.

The fast Rouse (higher order) and slow sticky Rouse (lower order) model contributions to the dynamic moduli are shown by dashed and dashed-dot-dashed lines in Figure 10. The sticky Rouse parameters are tabulated in Table 2. Since the sticky Rouse model does not take into account the glass mode relaxation, the Kohlrausch-Williams-Watts (KWW) model has been included to account for the glassy part of the dynamic rheological spectra. The Fourier transform of KWW model is given as,

$$
\begin{aligned}
& G_{K W W}^{\prime}(\omega)=\omega G_{g} \int_{0}^{\infty} \exp \left(-\left[t / \tau_{K W W}\right]^{\beta}\right) \sin (\omega t) d t \\
& G_{K W W}^{\prime \prime}(\omega)=\omega G_{g} \int_{0}^{\infty} \exp \left(-\left[t / \tau_{K W W}\right]^{\beta}\right) \cos (\omega t) d t
\end{aligned}
$$

where $G_{g}$ is the glassy modulus, $\beta$ is the stretch parameter of the exponential function, and $\tau_{K W W}$ defines the characteristic time of glassy relaxation. The empirical KWW fit is shown by dotted lines in Figure 12. The three fitting parameters i.e., $G_{g}, \beta$, and $\tau_{K W W}$ are tabulated in Table 3 of SI. Modes larger than a Kuhn segment is evidenced in the glassyrubbery transition where the KWW model deviates from the experimental data. The corresponding modulus $\sim 15 \mathrm{MPa}$ at deviation between experiment and theory gives an 
estimation of $M_{0}=200 \mathrm{~g} / \mathrm{mol}$, the Kuhn segment molecular weight. $\tau_{R, 0}$ is another parameter obtained from LVE, which roughly corresponds to the high frequency crossover and is different for the three samples because of different $T_{g}$. This completes the list of parameters to predict the experimental data using the sticky Rouse model described above.

Table 2: Parameters used in the sticky Rouse model

\begin{tabular}{ccccc} 
Sample & \multicolumn{2}{c}{ Rouse } & \multicolumn{2}{c}{ Sticky Rouse } \\
PMEA & $\tau_{R, 0}(\mathrm{~s})$ & $M_{0}(\mathrm{kDa})$ & $\tau_{s}^{*}(\mathrm{~s})$ & $M_{s}{ }^{a}(\mathrm{kDa})$ \\
P(MEA-co-UPy) $3 \%$ & $1 \times 10^{-9}$ & 0.2 & - & - \\
P(MEA-co-UPy) $8 \%$ & $4.5 \times 10^{-8}$ & 0.2 & 0.01 & 12.9 \\
${ }^{a}$ estimated by combining results from ${ }^{1} \mathrm{H}$ NMR spectroscopy and SEC.
\end{tabular}

In general there are three effects of increasing interaction strength and number of associations per chain on linear viscoelasticity: (i) the longest relaxation time increases, (ii) the plateau modulus increases, and (iii) the power law scaling in the terminal regime deviates towards lower magnitudes. ${ }^{6}$ Seiffert hypothesizes that (iii) is predominately due to polydispersity in the dispersion of associating groups along the backbone and not polydispersity in polymer molecular weight. ${ }^{44}$ Figure 10a-c shows the best fit model prediction via Eqs. 6 and 7 considering a monodisperse polymer molecular weight $M_{1}=\bar{M}_{w}$ measured from SEC. It is evident from Figures 10a-c that the model does an excellent job at predicting the shape of the experimental curve for timescales shorter than the longest relaxation time. For example, the model captures the plateau modulus quantitatively in Figures 10b-c. However the model does a poor job of predicting the experimental data for longer timescales. In Figure 10a, which has no hydrogen bonding groups present, the discrepency between experiment and model is hypothesized to be explicitly due to effects of polydispersity in molecular weight. 

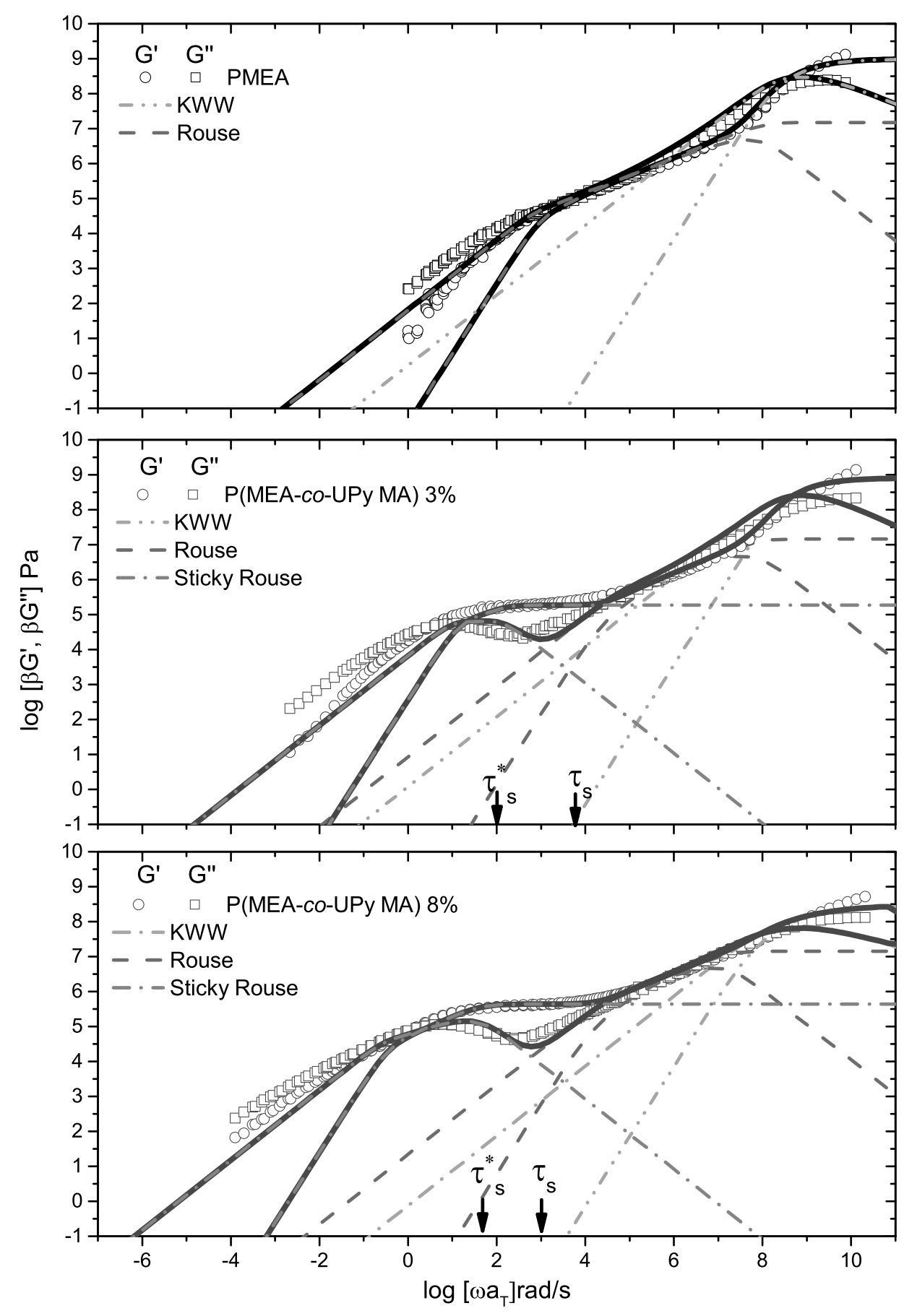

Figure 10: linear viscoelastic master curves of PMEA and P(MEA-co-UPyMA) systems shifted to $T_{r e f}=60{ }^{\circ} \mathrm{C}$. and the model prediction (solid lines) considering a monodisperse molecular weight.

The effect of polydispersity in molecular weight can be easily incorporated into the sticky Rouse model by discretizing the SEC molecular weight distribution and using Eqs 
6 and 7 as explained above. The molecular weight probability distribution function (pdf) from SEC was discretized into 540 points. Figures 11a-c (solid lines) show the model predictions taking into account MW polydispersity only. It is evident by comparing Figure 10a and Figure 11a that polydispersity in molecular weight does not account for the discrepancy between model and data. For the case of pure PMEA, the inability of the model to capture the data quantitatively could be due to the presence of entanglements, which are not explicitly taken into account in the model. Furthermore, there is divergence between model prediction and data for Figures 11b-c. For example, the predicted plateau modulus is lowered and the low frequency crossover is shifted to higher frequency. This observed divergence between model and data is explained by noting that the polydispersity model will statistically favor the more prevalent lower molecular weight species (lower modulus and higher crossover frequency), while the calculations made in Figure 10a-c uses only $\bar{M}_{w}$ which is skewed by larger molecular weight species. Moreover, this trend could be explained from the fact that SEC most likely gives an artificially lower average MW since a proper standard for calibration is not available for polymers with associating groups. For example, if the polymer interacts with the column via some association kinetics, then retention is delayed and the MW compared to PMMA standards would appear artificially small. The inclusion of polydispersity however, clearly shows a shallowing of the power-law scaling for low frequency due to the presence of large molecular weight species with larger number of sticky groups. This suggest that improved agreement between model and data must consider the distribution in number of sticky groups for a given MW. 

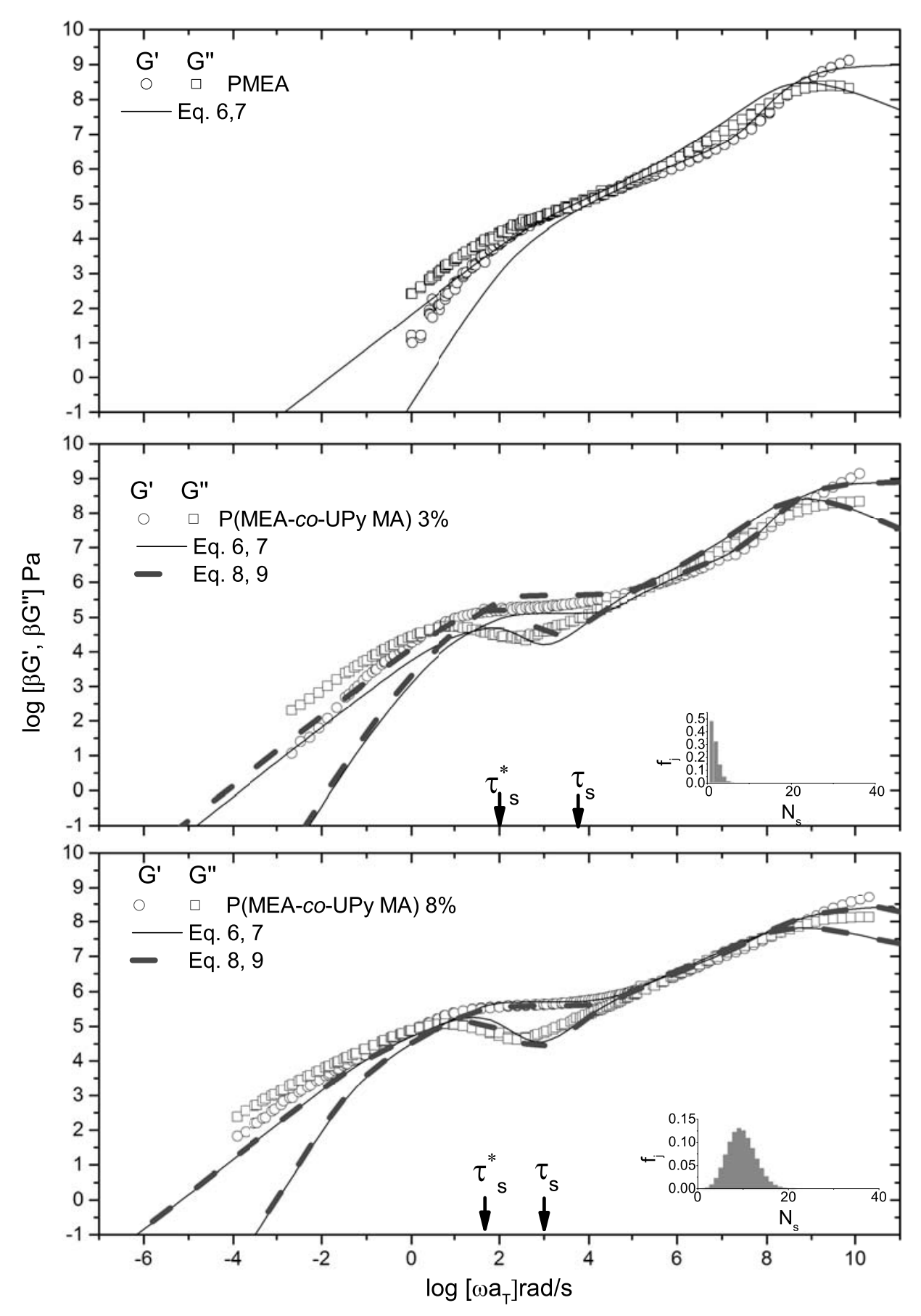

Figure 11: linear viscoelastic master curves of PMEA and P(MEA-co-UPyMA) systems shifted to $T_{r e f}=60{ }^{\circ} \mathrm{C}$. and the model prediction (solid lines) considering a polydispersity effects in MW as measured from SEC and (dotted lines) considering polydispersity effects in $\mathrm{MW}$ and $N_{s, j}$ via a binomial distirbution. Inset in b.) and c.): Binomial distribution function considering $\bar{M}_{w}$ from SEC.

We thus hypothesize that the inclusion of polydispersity in the number of sticky groups 
per chain will lead to close agreement of the model with the experimental data. Using Eqs. 9 and 10, we now consider the sticky Rouse model taking into account polydispersity in the number of stickers per chain via a binomial distribution as discussed above. The results are shown in Figures 11b-c (dashed lines). It is evident in the 3\% UPy case that the inclusion of a distribution in the number of stickers has stretched the model's low frequency crossover closer to the experimental data. However, it is evident that a distribution of stickers considering a binomial distribution cannot fully account for the low frequency response of the sample. Additionally, we find that in the 8\% UPy case the result considering a distribution in $N_{s}$ is almost identical to the result considering only polydispersity in MW. In fact, the two curves are almost indistinguishable. The insets in Figures $11 \mathrm{~b}-\mathrm{c}$ show the calculated binomial distribution considering the $\bar{M}_{w}$ determined from SEC. Note, there are several arguments as to why the binomial distribution may not be applicable, such as the assumed constant probability of monomer addition for the entire reaction. The binomial distribution may not be the accurate distribution in the case of the free radical polymerization carried out in this work.

One can speculate that the reduced power-law dependence of the moduli is due to the presence of a small number of molecules with a large number of stickers. In other words, the distribution of $N_{s}$ for a given MW follows a skewed distribution tending to large numbers. To generate such a distribution, we generate a normal distribution for $M_{s, j}$ around the mean value $M_{s}$ determined from NMR and SEC. We then generate a discrete distribution on the number of stickers via

$$
N_{s, j}=\frac{M_{i}}{M_{s, j}}
$$

The resulting distribution is shown in the insets of Figures 12a-b for the mean $\bar{M}_{w}$ determined from SEC. The parameters used to generate the distributions are $\sigma(3 \%)=4.5 \mathrm{kDa}$ $M_{s}=12.9 \mathrm{kDa}$ and $\sigma(8 \%)=3 \mathrm{kDa} M_{s}=6.4 \mathrm{kDa}$ with the constraint that $M_{s, j}>M_{m}$ 
(where $M_{m}$ is the molecular weight of one repeat unit). The distribution calculated above is coupled with Eqs. 9 and 10 to predict the experimental data. Comparison of model predictions and experimental data are shown in Figure 12a-b.

It is evident from Figures 12a-b that there is improved agreement of the model with experimental data. Specifically, the model more readily captures the shallowing of the low frequency power-law when a skewed distribution in $N_{s}$ is considered. This strongly suggests that a small number of molecules with a large number of stickers can strongly affect the low frequency response of the melt. In the case of chemistry where a distribution in $N_{s}$ are irrelevant, such as in the work performed considering ionic associating polymers where the spacing was highly controlled, the sticky Rouse model of Eqs. 6 and 7 showed excellent agreement for the entire LVE. ${ }^{1}$ It would appear that when the spacing of the associating groups are not highly controlled, a distribution in $N_{S}$ smears the low frequency behavior to lower power-laws, which must be taken into account when modeling such as in Eqs. 9 and 10. Overall the model does an excellent job of capturing the observed changes in LVE with increasing degree of association group density. These results suggest that indeed changes in LVE with association group density appear to be universal regardless of associating chemistry (e.g. ionic, hydrogen, etc.) 


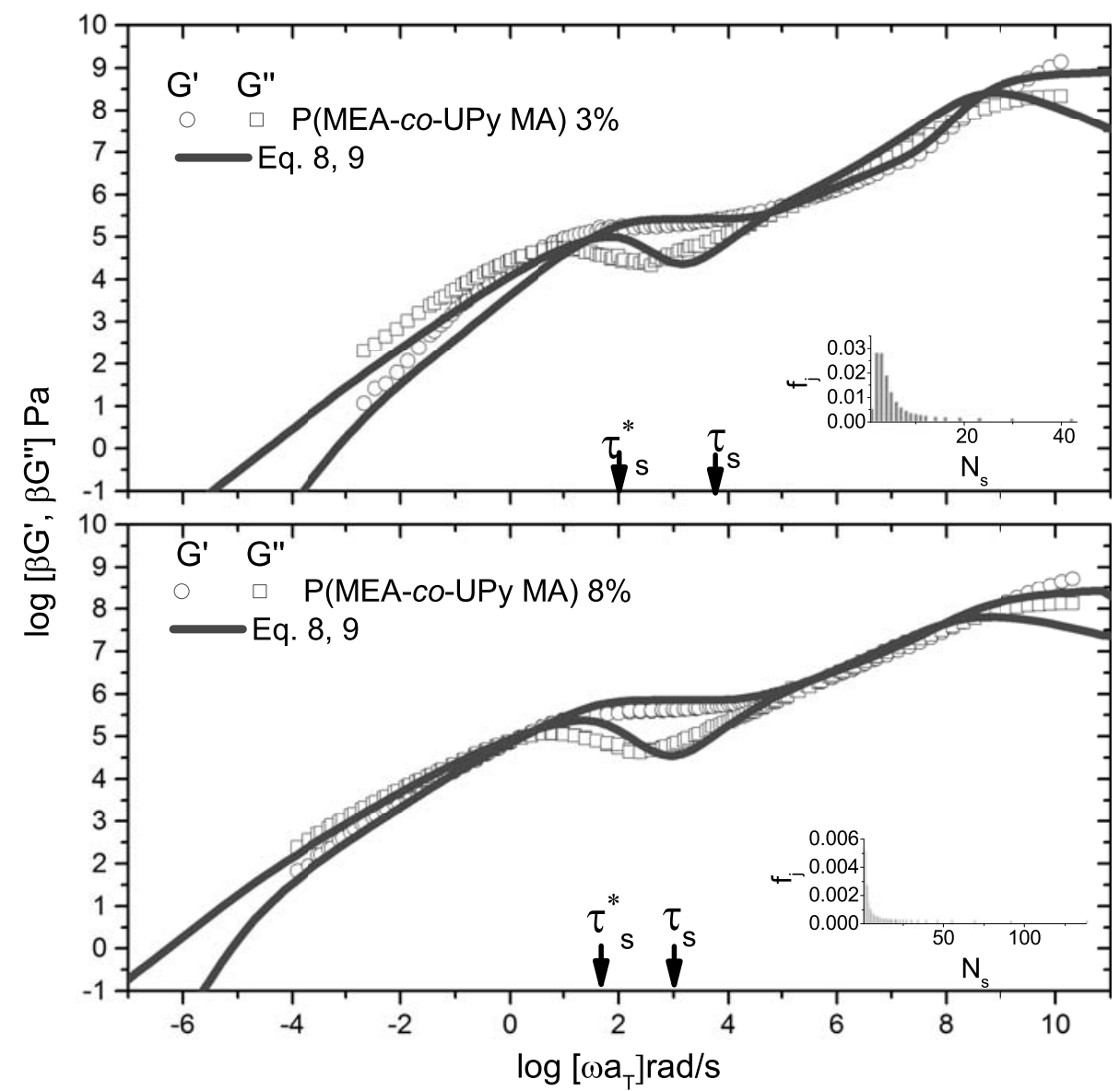

Figure 12: linear viscoelastic master curves of P(MEA-co-UPyMA) systems shifted to $T_{r e f}=60{ }^{\circ} \mathrm{C}$. and the model prediction (solid lines) considering both polydispersity in MW and a skewed distribution of $N_{s, j}$. Inset: Skewed distribution function of $N_{s}$ for $\bar{M}_{w}$.

\section{Conclusions}

We present a detailed study of the linear viscoelastic and DRS response of a series of unentangled linear polymers: PMEA, P(MEA-co-UPyMA) containing 3\% UPy, and P(MEAco-UPyMA) containing 8\% UPy, with approximately the same molecular weight. Linear viscoelasticity clearly shows the formation of hydrogen bonding polymer networks with a distinct plateau modulus, an increase in the terminal relaxation timescale, and a shallowing of the low frequency power-law dependence. DRS was used as an independent (non-rheological) technique to measure the association lifetime of hydrogen bond units. 
The timescale obtained from DRS represents a bare lifetime of hydrogen bond units which is order $k_{B} T$ per sticker. The timescale observed in rheology, the effective sticker lifetime, scales very well with the bare sticker lifetime, but is an order of magnitude larger than that measured via DRS. We argue that the difference is due to decreased probability of sticker disassociation above the gel point. The sticky Rouse model using parameters extracted from experimental data was predicted and compared to $G^{\prime}$ and $G^{\prime \prime}$. It is evident from the comparison that the sticky Rouse model accounting for polydispersity in MW is not sufficient in capturing the experimental trends. However, a modified sticky Rouse model where a distribution in $N_{S}$ is taken into account does an excellent job of capturing the low frequency power-law dependence. Furthermore, these results strongly suggest that a small number of chains with a large number of stickers can account for a lowering of the power-law scaling in the expected terminal regime. Overall, the comparison of model and experimental data suggest and support the hypothesis that the linear viscoelastic response of unentangled polymers is not unique to a given type of bond association. In other words, the associating bond chemistry does not have a profound effect on the linear viscoelastic response; allowing for the same theoretical analysis regardless of associating chemistry.

\section{Acknowledgement}

The work leading to these results has received funding from the People Programme (Marie Curie Actions) of the European Union's Seventh Framework Programme (FP7/20072013) under REA grant agreement no. 607937 and and the Danish National Advanced Technology Foundation (grant no. 55-2013-3).

\section{Supporting Information Available}

This material is available free of charge via the Internet at http://pubs . acs . org/. 


\section{References}

(1) Chen, Q.; Tudryn, G. J.; Colby, R. H. Ionomer dynamics and the sticky Rouse model a ). J. Rheol. 2013, 16802, 1441-1462.

(2) Chen, Q.; Masser, H.; Shiau, H.-S.; Liang, S.; Runt, J.; Painter, P. C.; Colby, R. H. Linear Viscoelasticity and Fourier Transform Infrared Spectroscopy of Polyether-Ester-Sulfonate Copolymer Ionomers. Macromolecules 2014, 47, 36353644 .

(3) Ling, G. H.; Wang, Y.; Weiss, R. A. Linear Viscoelastic and Uniaxial Extensional Rheology of Alkali Metal Neutralized Sulfonated Oligostyrene Ionomer Melts. Macromolecules 2012, 45, 481-490.

(4) Stadler, F. J.; Pyckhout-Hintzen, W.; Schumers, J.-M.; Fustin, C.-A.; Gohy, J.-F.; Bailly, C. Linear Viscoelastic Rheology of Moderately Entangled Telechelic Polybutadiene Temporary Networks. Macromolecules 2009, 42, 6181-6192.

(5) González, L.; Ladegaard Skov, A.; Hvilsted, S. Ionic networks derived from the protonation of dendritic amines with carboxylic acid end-functionalized PEGs. J. Polym. Sci. Polym. Chem. 2013, 51, 1359-1371.

(6) Shabbir, A.; Goldansaz, H.; Hassager, O.; van Ruymbeke, E.; Alvarez, N. J. Effect of Hydrogen Bonding on Linear and Nonlinear Rheology of Entangled Polymer Melts. Macromolecules 2015, 48, 5988-5996.

(7) Kumpfer, J. R.; Wie, J. J.; Swanson, J. P.; Beyer, F. L.; Mackay, M. E.; Rowan, S. J. Influence of Metal Ion and Polymer Core on the Melt Rheology of Metallosupramolecular Films. Macromolecules 2012, 45, 473-480.

(8) Rossow, T.; Seiffert, S. Supramolecular polymer gels with potential model-network structure. Polym. Chem. 2014, 5, 3018-3029. 
(9) Bosman, A. W.; Sijbesma, R. P.; Meijer, E. W. Supramolecular polymers at work. Mater. Today 2004, 7, 34-39.

(10) Tang, S.; Wang, M.; Olsen, B. D. Anomalous self-diffusion and sticky Rouse dynamics in associative protein hydrogels. J. Am. Chem. Soc. 2015, 137, 3946-57.

(11) Lewis, C. L.; Stewart, K.; Anthamatten, M. The Influence of Hydrogen Bonding SideGroups on Viscoelastic Behavior of Linear and Network Polymers. Macromolecules 2014, 47, 729-740.

(12) Feldman, K. E.; Kade, M. J.; Meijer, E. W.; Hawker, C. J.; Kramer, E. J. Model Transient Networks from Strongly Hydrogen-Bonded Polymers. Macromolecules 2009, 42, 90729081.

(13) Rubinstein, M.; Semenov, A. N. Dynamics of Entangled Solutions of Associating Polymers. Macromolecules 2001, 34, 1058-1068.

(14) Baxandall, L. G. Dynamics of Reversibly Cross-Linked Chains. Macromolecules 1989, 22, 1982-1988.

(15) Green, M. S.; Tobolsky, A. V. A New Approach to the Theory of Relaxing Polymeric Media. J. Chem. Phys. 1946, 14, 80-92.

(16) Rubinstein, M.; Semenov, A. N. Thermoreversible Gelation in Solutions of Associating Polymers. 2. Linear Dynamics. Macromolecules 1998, 31, 1386-1397.

(17) Leibler, L.; Rubinstein, M.; Colby, R. H. Dynamics of Reversible Networks. Macromolecules 1991, 24, 4701-4707.

(18) Sijbesma, R. P.; Beijer, F. H.; Brunsveld, L.; Folmer, B. J. B.; Hirschberg, J. H. K. K. Reversible Polymers Formed from Self-Complementary Monomers Using Quadruple Hydrogen Bonding. Science 1997, 278, 1601-1604. 
(19) Yamauchi, K.; Lizotte, J. R.; Long, T. E. Thermoreversible Poly ( alkyl acrylates ) Consisting of Self-Complementary Multiple Hydrogen Bonding. Macromolecules 2003, 36, 1083-1088.

(20) Elkins, C. L.; Park, T.; McKee, M. G.; Long, T. E. Synthesis and characterization of poly(2-ethylhexyl methacrylate) copolymers containing pendant, selfcomplementary multiple-hydrogen-bonding sites. J. Polym. Sci. Polym. Chem. 2005, $43,4618-4631$.

(21) Lewis, C. L.; Anthamatten, M. Synthesis, swelling behavior, and viscoelastic properties of functional poly(hydroxyethyl methacrylate) with ureidopyrimidinone sidegroups. Soft Matter 2013, 9, 4058.

(22) Tanaka, M.; Sato, K.; Kitakami, E.; Kobayashi, S.; Hoshiba, T.; Fukushima, K. Design of biocompatible and biodegradable polymers based on intermediate water concept. Polym. J. 2014, 47, 114-121.

(23) Tinius, J. A.; Dragomer, D.; Klutka, F.; Vanbebber, A.; Cerney, K. The Capiox RX05 oxygenator : pediatric clinical observations. Perfusion 2003, 18, 321-323.

(24) Javakhishvili, I.; Kasama, T.; Jankova, K.; Hvilsted, S. RAFT copolymerization of itaconic anhydride and 2-methoxyethyl acrylate: a multifunctional scaffold for preparation of "clickable" gold nanoparticles. Chem. Commun. 2013, 49, 4803-4805.

(25) Javakhishvili, I.; Jankova, K.; Hvilsted, S. Neutral, anionic, cationic, and zwitterionic diblock copolymers featuring poly(2-methoxyethyl acrylate) "hydrophobic" segments. Polym. Chem. 2013, 4, 662-668.

(26) Røn, T.; Javakhishvili, I.; Patil, N. J.; Jankova, K.; Zappone, B.; Hvilsted, S.; Lee, S. Aqueous lubricating properties of charged ( $\mathrm{ABC}$ ) and neutral (ABA) triblock copolymer chains. Polymer 2014, 55, $4873-4883$. 
(27) Javakhishvili, I.; Tanaka, M.; Ogura, K.; Jankova, K.; Hvilsted, S. Synthesis of Graft Copolymers Based on Poly ( 2- Methoxyethyl Acrylate ) and Investigation of the Associated Water Structure. Macromol. Rapid Commun. 2012, 319-325.

(28) Javakhishvili, I.; Røn, T.; Jankova, K.; Hvilsted, S.; Lee, S. Synthesis, Characterization, and Aqueous Lubricating Properties of Amphiphilic Graft Copolymers Comprising 2-Methoxyethyl Acrylate. Macromolecules 2014, 47, 2019-2029.

(29) Schröter, K.; Hutcheson, S. A.; Shi, X.; Mandanici, A.; McKenna, G. B. Dynamic shear modulus of glycerol: Corrections due to instrument compliance. J. Chem. Phys. 2006, $125,214507$.

(30) de Greef, T. F. A.; Nieuwenhuizen, M. M. L.; Sijbesma, R. P.; Meijer, E. W. Competitive intramolecular hydrogen bonding in oligo(ethylene oxide) substituted quadruple hydrogen bonded systems. J. Org. chem. 2010, 75, 598-610.

(31) Hackelbusch, S.; Rossow, T.; Assenbergh, P. V. Chain Dynamics in Supramolecular Polymer Networks. Macromolecules 2013, 46, 6273-6286.

(32) Lange, R. F. M.; Van Gurp, M.; Meijer, E. W. Hydrogen-bonded supramolecular polymer networks. J. Polym. Sci. Polym. Chem. 1999, 37, 3657-3670.

(33) Chen-Yang Liu, R. K., Jiasong He; Bailly, C. New Linearized Relation for the Universal Viscosity-Temperature Behavior of Polymer Melts. Macromolecules 2006, 39, 8867-8869.

(34) Noro, A.; Matsushita, Y.; Lodge, T. P. Thermoreversible Supramacromolecular Ion Gels via Hydrogen Bonding. Macromolecules 2008, 41, 5839-5844.

(35) McKee, M. G.; Elkins, C. L.; Park, T.; Long, T. E. Influence of Random Branching on Multiple Hydrogen Bonding in Poly(alkyl methacrylate)s. Macromolecules 2005, 38, 6015-6023. 
(36) Kremer, F.; Schönhals, A. Broadband Dielectric Spectroscopy; Springer Berlin Heidelberg, 2003.

(37) Wübbenhorst, M.; van Turnhout, J. Analysis of complex dielectric spectra. I. Onedimensional derivative techniques and three-dimensional modelling. J. Non-Cryst. Solids 2002, 305, $40-49$.

(38) Cole, K. S.; Cole, R. H. Dispersion and Absorption in Dielectrics II. Direct Current Characteristics. J. Chem. Phys. 1942, 10.

(39) Vogel, H. The law of the relationship between viscosity of liquids and the temperature. Phys. Z 1921, 22, 645-646.

(40) Fulcher, G. A. Analysis of recent measurements of the viscosity of glasses. J. Am. Ceram. Soc. 1925, 8, 339-355.

(41) Wubbenhorst, M.; Turnhout, J. V.; Folmer, B. J. B.; Sijbesma, R. P.; Meijer, E. W. Complex Dynamics of Hydrogen Bonded. IEEE Trans. Dielectr. Electr. Insul. 2001, 8, 365372.

(42) Lou, N.; Wang, Y.; Li, X.; Li, H.; Wang, P.; Wesdemiotis, C.; Sokolov, A. P.; Xiong, H. Dielectric Relaxation and Rheological Behavior of Supramolecular Polymeric Liquid. Macromolecules 2013, 46, 3160-3166.

(43) Ferry, J. Viscoelastic Properties of Polymers, 3rd ed.; Wiley, New York, 1980.

(44) Seiffert, S. Effect of Supramolecular Interchain Sticking on the Low-Frequency Relaxation of Transient Polymer Networks. Macromol. Rapid Commun. 2016, 37, 257-264.

(45) Chen, Q.; Huang, C.; Weiss, R. a.; Colby, R. H. Viscoelasticity of Reversible Gelation for Ionomers. Macromolecules 2015, 48, 1221-1230. 